\title{
On the Multipeakon Dissipative Behavior of the Modified Coupled Camassa-Holm Model for Shallow Water System
}

\author{
Zhixi Shen, ${ }^{1}$ Yujuan Wang, ${ }^{1}$ Hamid Reza Karimi, ${ }^{2}$ and Yongduan Song ${ }^{1}$ \\ ${ }^{1}$ School of Automation, Chongqing University, Chongqing 400044, China \\ ${ }^{2}$ Department of Engineering, Faculty of Technology and Science, University of Agder, N-4898 Grimstad, Norway
}

Correspondence should be addressed to Yongduan Song; ydsong@cqu.edu.cn

Received 9 May 2013; Accepted 26 June 2013

Academic Editor: Hongli Dong

Copyright (c) 2013 Zhixi Shen et al. This is an open access article distributed under the Creative Commons Attribution License, which permits unrestricted use, distribution, and reproduction in any medium, provided the original work is properly cited.

\begin{abstract}
This paper investigates the multipeakon dissipative behavior of the modified coupled two-component Camassa-Holm system arisen from shallow water waves moving. To tackle this problem, we convert the original partial differential equations into a set of new differential equations by using skillfully defined characteristic and variables. Such treatment allows for the construction of the multipeakon solutions for the system. The peakon-antipeakon collisions as well as the dissipative behavior (energy loss) after wave breaking are closely examined. The results obtained herein are deemed valuable for understanding the inherent dynamic behavior of shallow water wave breaking.
\end{abstract}

\section{Introduction}

The study of the dynamic behavior of shallow water wave represents an important research topic in view of its potential application in surface and underwater vehicle systems design, control, deployment, and monitoring. There are several classical models describing the motion of waves at the free surface of shallow water under the influence of gravity, the best known of which are the Korteweg-de Vries (KdV) equation $[1,2]$ and the Camassa-Holm $(\mathrm{CH})$ equation [3-5]. The KdV equation admits solitary wave solutions but does not model the phenomenon of breaking for water waves. The $\mathrm{CH}$ equation, modeling the unidirectional propagation of shallow water waves over a flat bottom [4-6] as well as water waves moving over an underlying shear flow [7], has many remarkable properties like solitary waves with singularities called peakons $[4,6]$ and breaking waves $[4,8]$ which set it apart from $\mathrm{KdV}$. The peaked solitary waves mean that they are smooth except at the crests, where they are continuous but have a jump discontinuity in the first derivative, while the presence of breaking waves means that the solution remains bounded while its slope becomes unbounded in finite time $[8,9]$. After wave breaking the solutions of the $\mathrm{CH}$ equation, as shown by several works [10-16], become uniquely as either global conservative or global dissipative solutions.
Recently, the $\mathrm{CH}$ equation has been extended to many multicomponent generalizations, which can better reflect the feature of the shallow water moving. In this paper, we consider the following modified coupled two-component Camassa-Holm system [17]:

$$
\begin{gathered}
m_{t}+2 m u_{x}+m_{x} u+(m v)_{x}+n v_{x}=0, \quad t>0, x \in R, \\
n_{t}+2 n v_{x}+n_{x} v+(n u)_{x}+m u_{x}=0, \quad t>0, x \in R, \\
m=u-u_{x x}, \quad t>0, x \in R, \\
n=v-v_{x x}, \quad t>0, \quad x \in R,
\end{gathered}
$$

which is a modified version of the coupled two-component Camassa-Holm system as established by Fu and Qu in [18], allowing for peakon solitons in the form of a superposition of multipeakons. System (1) can be rewritten as a Hamiltonian system,

$$
\frac{\partial}{\partial t}\left(\begin{array}{c}
m \\
n
\end{array}\right)=-\left(\begin{array}{ll}
\partial m+m \partial & \partial m+n \partial \\
\partial n+m \partial & \partial n+n \partial
\end{array}\right)\left(\begin{array}{l}
\frac{\delta H}{\delta m}=u \\
\frac{\delta H}{\delta n}=v
\end{array}\right)
$$

with the Hamiltonian $H=(1 / 2) \int(m G * m+n G * n) d x$, where $G * m=u, G * n=v$, and $G=(1 / 2) e^{-|x|}$. Particularly, when 
$u=0$ (or $v=0$ ), the degenerated equation (1) has the same peakon solitons as the $\mathrm{CH}$ equation. We are interested in such system because it exhibits the following conserved quantities, as can be easily verified:

$$
\begin{array}{ll}
E_{1}(u)=\int_{R} u d x, & E_{2}(v)=\int_{R} v d x, \\
E_{3}(u)=\int_{R} m d x, & E_{4}(u)=\int_{R} n d x, \\
E_{5}(u, v)=\int_{R}\left(u^{2}+u_{x}^{2}+v^{2}+v_{x}^{2}\right) d x .
\end{array}
$$

It has been shown that system (1) is locally well-posed and also has global strong solutions which blow up in finite time [17, 18]. Moreover, the existence issue for a class of local weak solutions for such system was also addressed in [17]. It is interesting to know that whether the two remarkable properties associated with the original $\mathrm{CH}$ equation persist in this modified coupled two-component Camassa-Holm system. In our recent work [19, 20], we studied the problem of solution continuation beyond wave breaking of system (1), where it was established that the system admits either global conservative solutions or global dissipative solutions.

Just as with the $\mathrm{CH}$ equation, the multipeakon dissipative solution represents an important aspect related to the solutions near wave breaking, it is interesting to know whether or not system (1) also exhibits the similar feature. Thus far very little effort has been made on studying the multipeakon dissipative solution associated with the modified coupled two-component Camassa-Holm system of the form as expressed in (1) in the literature. Based on our recent work [20] where a global continuous semigroup of dissipative solutions of system (1) is established, in this paper we show how to construct globally defined multipeakon solutions in the dissipative case for the modified coupled twocomponent Camassa-Holm system.

It should be stressed that the system considered in this work is a heavily coupled one; it is the mutual effect between the two components that makes the analysis and computation much more involved than the system with single component as studied in [16]. The key to circumvent the difficulty is to utilize a skillfully defined characteristic and several new variables to obtain a new set of ordinary differential equations, from which the dissipative multipeakons are globally determined. Such feature discovered is deemed useful in further understanding the dynamic behavior of the wave breaking associated with the system. Examples are presented to illustrate the feature of the multipeakons with peakonantipeakon collisions.

The rest of this paper is organized as follows. Section 2 represents the construction of the global dissipative solutions of the modified coupled Camassa-Holm system. Section 3 is devoted to the establishment of the dissipative multipeakon solutions of system (1). The method is illustrated by explicit calculations in the case $n=1$ and by numerical computations when $n=2$ with peakon-antipeak on collisions in Section 4 . The paper is concluded in Section 5 .

\section{Global Dissipative Solutions of the Modified Coupled Camassa-Holm System}

We represent the construction of the global dissipative solutions of system (1) obtained in [20] in this section. System (1) can be rewritten as

$$
\begin{array}{ll}
u_{t}+(u+v) u_{x}+P_{1}+P_{2, x}=0, & t>0, x \in R, \\
v_{t}+(u+v) v_{x}+P_{3}+P_{4, x}=0, & t>0, x \in R,
\end{array}
$$

where $P_{1}, P_{2}, P_{3}, P_{4}$ are given by

$$
\begin{aligned}
P_{1}(t, x)= & G *\left(u v_{x}\right)=\frac{1}{2} \cdot \int_{R} e^{-\left|x-x^{\prime}\right|}\left(u v_{x}\right)\left(t, x^{\prime}\right) d x^{\prime}, \\
P_{2}(t, x)= & G *\left(u^{2}+\frac{u_{x}^{2}}{2}+u_{x} v_{x}+\frac{v^{2}}{2}-\frac{v_{x}^{2}}{2}\right) \\
= & \frac{1}{2} \cdot \int_{R} e^{-\left|x-x^{\prime}\right|}\left(u^{2}+\frac{u_{x}^{2}}{2}+u_{x} v_{x}+\frac{v^{2}}{2}-\frac{v_{x}^{2}}{2}\right) \\
& \times\left(t, x^{\prime}\right) d x^{\prime}, \\
P_{3}(t, x)= & G *\left(v u_{x}\right)=\frac{1}{2} \cdot \int_{R} e^{-\left|x-x^{\prime}\right|}\left(v u_{x}\right)\left(t, x^{\prime}\right) d x^{\prime}, \\
P_{4}(t, x)= & G *\left(v^{2}+\frac{v_{x}^{2}}{2}+u_{x} v_{x}+\frac{u^{2}}{2}-\frac{u_{x}^{2}}{2}\right) \\
= & \frac{1}{2} \cdot \int_{R} e^{-\left|x-x^{\prime}\right|}\left(v^{2}+\frac{v_{x}^{2}}{2}+u_{x} v_{x}+\frac{u^{2}}{2}-\frac{u_{x}^{2}}{2}\right) \\
& \times\left(t, x^{\prime}\right) d x^{\prime},
\end{aligned}
$$

with $G=e^{-|x|} / 2$ the Green's function such as $G * f(x)=$ $1 / 2 \cdot \int_{R} e^{-\left|x-x^{\prime}\right|} f\left(x^{\prime}\right) d x^{\prime}$ for all $f \in L^{2}(R)$ and $*$ the spatial convolution.

By using a skillfully defined characteristic $y_{t}(t, \xi)=(u+$ $v)(t, y(t, \xi))$, which can be decomposed as $y(t, \xi)=\varsigma(t, \xi)+\xi$, and a new set of Lagrangian variables; namely,

$$
\begin{gathered}
h(t, \xi)=\left(u^{2}+u_{x}^{2}+v^{2}+v_{x}^{2}\right) \circ y y_{\xi}, \\
U(t, \xi)=u(t, y(t, \xi)), \\
V(t, \xi)=v(t, y(t, \xi)), \\
M(t, \xi)=u_{x}(t, y(t, \xi)), \\
N(t, \xi)=v_{x}(t, y(t, \xi))
\end{gathered}
$$


where $h$ corresponds to the Lagrangian energy density and $U$, $V$ the Lagrangian velocity, we derive an equivalent system of the modified coupled Camassa-Holm system,

$$
\begin{gathered}
\varsigma_{t}=U+V, \quad U_{t}=-P_{1}-P_{2, x}, \\
V_{t}=-P_{3}-P_{4, x}, \\
M_{t}=\left(-\frac{M^{2}}{2}-\frac{N^{2}}{2}+U^{2}+\frac{V^{2}}{2}-P_{1, x}-P_{2}\right), \\
N_{t}=\left(-\frac{N^{2}}{2}-\frac{M^{2}}{2}+V^{2}+\frac{U^{2}}{2}-P_{3, x}-P_{4}\right), \\
h_{t}=\left(3 U^{2}-2 P_{2}\right) U_{\xi}-2 U P_{2, x} y_{\xi} \\
+\left(3 V^{2}-2 P_{4}\right) V_{\xi}-2 V P_{4, x} y_{\xi} .
\end{gathered}
$$

It is shown in [20] that the existence, uniqueness, and stability of solutions of system (7) are obtained in a Banach space, which is transformed into the conservative solution of the original system (4), while dissipative solutions differ from conservative solutions when particles collide, that is, when $y_{\xi}(t, \xi)=0$ for $\xi$ in an interval of positive length. To obtain the dissipative solution, we impose that when particles collide, they lose their energy; that is, if $y_{\xi}(\tau, \xi)=0$ for some $\tau$, then we set $h(\tau, \xi)=0$. Thus we define $\tau(\xi)$ as the first time when $y_{\xi}(t, \xi)$ vanishes; namely,

$$
\tau(\xi)=\sup \left\{t \in R^{+} \mid y_{\xi}\left(t^{\prime}, \xi\right)>0 \forall 0 \leq t^{\prime}<t\right\},
$$

and the expressions for $P_{i}$ and $P_{i, x}(i=1,2,3,4)$ become

$$
\begin{aligned}
& P_{1}(t, \xi)=\frac{1}{2} \cdot \int_{t<\tau(\xi)} e^{-\left|y(\xi)-y\left(\xi^{\prime}\right)\right|} \\
& \times\left[(U N) y_{\xi}\right]\left(\xi^{\prime}\right) d \xi^{\prime}, \\
& P_{1, x}(t, \xi)=-\frac{1}{2} \cdot \int_{t<\tau(\xi)} \operatorname{sgn}\left(\xi-\xi^{\prime}\right) e^{-\left|y(\xi)-y\left(\xi^{\prime}\right)\right|} \\
& \times\left[(U N) y_{\xi}\right]\left(\xi^{\prime}\right) d \xi^{\prime}, \\
& P_{2}(t, \xi)=\frac{1}{4} \cdot \int_{t<\tau(\xi)} e^{-\left|y(\xi)-y\left(\xi^{\prime}\right)\right|} \\
& \times\left[h+\left(U^{2}+2 M N-N^{2}\right) y_{\xi}\right] \\
& \times\left(\xi^{\prime}\right) d \xi^{\prime}, \\
& P_{2, x}(t, \xi)=-\frac{1}{4} \cdot \int_{t<\tau(\xi)} \operatorname{sgn}\left(\xi-\xi^{\prime}\right) e^{-\left|y(\xi)-y\left(\xi^{\prime}\right)\right|} \\
& \times\left[h+\left(U^{2}+2 M N-N^{2}\right) y_{\xi}\right] \\
& \times\left(\xi^{\prime}\right) d \xi^{\prime},
\end{aligned}
$$

$$
\begin{gathered}
P_{3}(t, \xi)=\frac{1}{2} \cdot \int_{t<\tau(\xi)} e^{-\left|y(\xi)-y\left(\xi^{\prime}\right)\right|} \\
\times\left[(V M) y_{\xi}\right]\left(\xi^{\prime}\right) d \xi^{\prime}, \\
\begin{aligned}
P_{3, x}(t, \xi)=-\frac{1}{2} \cdot \int_{t<\tau(\xi)} \operatorname{sgn}\left(\xi-\xi^{\prime}\right) e^{-\left|y(\xi)-y\left(\xi^{\prime}\right)\right|} \\
\times\left[(V M) y_{\xi}\right]\left(\xi^{\prime}\right) d \xi^{\prime}, \\
P_{4}(t, \xi)=\frac{1}{4} \cdot \int_{t<\tau(\xi)} e^{-\left|y(\xi)-y\left(\xi^{\prime}\right)\right|} \\
\times\left[h+\left(V^{2}+2 M N-M^{2}\right) y_{\xi}\right] \\
\times\left(\xi^{\prime}\right) d \xi^{\prime}, \\
P_{4, x}(t, \xi)=-\frac{1}{4} \cdot \int_{t<\tau(\xi)} \operatorname{sgn}\left(\xi-\xi^{\prime}\right) e^{-\left|y(\xi)-y\left(\xi^{\prime}\right)\right|} \\
\times\left[h+\left(V^{2}+2 M N-M^{2}\right) y_{\xi}\right] \\
\times\left(\xi^{\prime}\right) d \xi^{\prime},
\end{aligned}
\end{gathered}
$$

and the modified system to be solved here reads

$$
\begin{gathered}
\varsigma_{t}=U+V, \quad U_{t}=-P_{1}-P_{2, x}, \\
V_{t}=-P_{3}-P_{4, x},
\end{gathered}
$$$$
M_{t}=\left(-\frac{M^{2}}{2}-\frac{N^{2}}{2}+U^{2}+\frac{V^{2}}{2}-P_{1, x}-P_{2}\right) \text {, }
$$

$$
N_{t}=\left(-\frac{N^{2}}{2}-\frac{M^{2}}{2}+V^{2}+\frac{U^{2}}{2}-P_{3, x}-P_{4}\right) \text {. }
$$$$
\varsigma_{\xi t}= \begin{cases}U_{\xi}+V_{\xi} & \text { if } t<\tau(\xi), \\ 0 & \text { otherwise, }\end{cases}
$$$$
U_{\xi t}=\left\{\begin{aligned}
\frac{h}{2}+\left(\frac{U^{2}}{2}+M N-N^{2}\right. & \\
\left.-P_{2}-P_{1, x}\right) y_{\xi} & \text { if } t<\tau(\xi), \\
0 & \text { otherwise, }
\end{aligned}\right.
$$$$
V_{\xi t}=\left\{\begin{aligned}
\frac{h}{2}+\left(\frac{V^{2}}{2}+M N-M^{2}\right. & \\
\left.-P_{4}-P_{3, x}\right) y_{\xi} & \text { if } t<\tau(\xi), \\
0 & \text { otherwise }
\end{aligned}\right.
$$

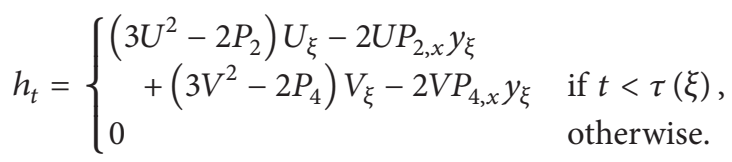


Note that, in this definition, we do not reset the energy density $h$ to zero for $t \geq \tau(\xi)$ but keep the value it reached just before the collision, which has the advantage of rendering the right hand of (11) continuous across the value $t=\tau(\xi)$ and the behavior of the system remains unchanged.

The local existence of solutions is proved in the Banach space $E$ where

$$
\begin{gathered}
E=L^{\infty} \cap W \cap W \cap W \cap W \cap W \cap W \cap W \cap L^{1}, \\
W=L^{2} \cap L^{\infty} .
\end{gathered}
$$

The global solutions of (10) may not exist for all initial data in $E$; however, they exist when the initial data $X_{0}=$ $\left(\varsigma_{0}, U_{0}, V_{0}, M_{0}, N_{0}, h_{0}\right) \in \Gamma$, where $\Gamma$ is defined as follows.

Definition 1. The set $\Gamma$ is composed of all $(\varsigma, U, V, M, N, h)$ such that

$$
\begin{gathered}
X=\left(\varsigma, U, V, M, N, \varsigma_{\xi}, U_{\xi}, V_{\xi}, h\right) \in E, \\
y_{\xi} \geq 0, \quad h \geq 0 \text { almost everywhere, } \\
y_{\xi} h=y_{\xi}^{2} U^{2}+U_{\xi}^{2}+y_{\xi}^{2} V^{2}+V_{\xi}^{2}, \text { almost everywhere, } \\
\frac{1}{\left(y_{\xi}+h\right)} \in L^{\infty}(R), \\
g\left(y, U, V, y_{\xi}, U_{\xi}, V_{\xi}, h\right)-1 \in W
\end{gathered}
$$

where $g(\mathbf{x})$ is given by

$$
g(\mathbf{x})= \begin{cases}\left|x_{5}\right|+\left|x_{6}\right|+2\left(1+x_{2}^{2}+x_{3}^{2}\right) x_{4}, & \text { if } x \in \Omega \\ x_{4}+x_{7}, & \text { otherwise }\end{cases}
$$

for $\mathbf{x}=\left(x_{1}, x_{2}, x_{3}, x_{4}, x_{5}, x_{6}, x_{7}\right) \in R^{7}$, where $\Omega$ is the following subset of $R^{7}$

$$
\begin{gathered}
\Omega=\left\{\mathbf{x} \in R^{7}|| x_{5}|+| x_{6} \mid+2\left(1+x_{2}^{2}+x_{3}^{2}\right)\right. \\
\left.\times x_{4} \leq x_{4}+x_{7}, x_{5}, x_{6} \leq 0\right\} .
\end{gathered}
$$

The main result in [20] is stated in the following theorem.

Theorem 2. Let $z_{0}=\left(u_{0}, v_{0}\right) \in H^{1} \times H^{1}$ be given . If one denotes $t \rightarrow z(t)=T_{t}\left(z_{0}\right)$ the corresponding trajectory, then $z=(u, v)$ is a weak dissipative solution of the modified coupled two-component Camassa-Holm system, which constructs a continuous semigroup with respect of the metric $d_{H^{1}}$ on bounded sets of $H^{1}$; that is, for any $M>0$ and any sequence $z_{n} \in H^{1}$ such that $\left\|z_{n}\right\|_{H^{1}} \leq M$, one has that $\lim _{n \rightarrow \infty} d_{H^{1}}\left(z_{n}, z\right)=0$ implies $\lim _{n \rightarrow \infty} d_{H^{1}}\left(T_{t}\left(z_{n}\right), T_{t}(z)\right)=$ 0 .

\section{Multipeakon Dissipative Solutions of the Original System}

In this section, we derive a new system of ordinary differential equations for the multipeakon solutions which is well-posed even when collisions occur, and the variables $(y, U, V, M, N, H)$ are used to characterize multipeakons in a way that avoids the problems related to blowing up.

Solutions of the modified coupled two-component Camassa-Holm system may experience wave breaking in the sense that the solution develops singularities in finite time, while keeping the $H^{1}$ norm finite. Continuation of the solution beyond wave breaking imposes significant challenge as can be illustrated in the case of multipeakons, which are special solutions of the modified coupled two-component Camassa-Holm system of the form

$$
(u, v)(t, x)=\left(\sum_{i=1}^{n} p_{i}(t) e^{-\left|x-q_{i}(t)\right|}, \sum_{i=1}^{n} r_{i}(t) e^{-\left|x-q_{i}(t)\right|}\right),
$$

where $\left(p_{i}(t), r_{i}(t), q_{i}(t)\right)$ satisfy the explicit system of ordinary differential equations

$$
\begin{gathered}
\dot{p}_{i}=\sum_{j=1, i \neq j}^{n}\left(p_{i} p_{j}+r_{i} r_{j}\right) \operatorname{sgn}\left(q_{j}-q_{i}\right) e^{-\left|q_{i}-q_{j}\right|}, \\
\dot{r}_{i}=\sum_{j=1, i \neq j}^{n}\left(p_{i} p_{j}+r_{i} r_{j}\right) \operatorname{sgn}\left(q_{j}-q_{i}\right) e^{-\left|q_{i}-q_{j}\right|}, \\
\dot{q}_{i}=-\sum_{j=1}^{n}\left(p_{j}+r_{j}\right) e^{-\left|q_{i}-q_{j}\right|} .
\end{gathered}
$$

Let us consider initial data $z_{0}=\left(u_{0}, v_{0}\right)$ given by

$$
\left(u_{0}, v_{0}\right)(x)=\left(\sum_{i=1}^{n} p_{i} e^{-\left|x-\xi_{i}\right|}, \sum_{i=1}^{n} r_{i} e^{-\left|x-\xi_{i}\right|}\right) .
$$

Peakons interact in a way similar to that of solitons of the $\mathrm{CH}$ equation, and wave breaking may appear when at least two of the $q_{i}$ coincide. In the case that $p_{i}(0)$ and $r_{i}(0)$ have the same sign for all $i=1,2, \ldots n$ and $q_{i}(t)$ remain distinct, (21) allows for a unique global solution, where the peakons are traveling in the same direction. By inserting that solution into $(20)$, it is not hard to know that $z=(u, v)$ is a global weak solution of system (4). However, when two peakons have opposite signs, collisions may occur, and if so, the system (21) blows up. Without loss of generality, we assume that the $p_{i}$ and $r_{i}$ are all nonzero, and that the $\xi_{i}$ are all distinct. The aim is to characterize the unique and global weak solution from Theorem 2 with initial data (22) explicitly. Since the variables $p_{i}$ and $r_{i}$ blow up at collisions, they are not appropriate to define a multipeakon in the form of (20). We consider the following characterization of multipeakons given as continuous solutions $z=(u, v)$, which are defined on intervals $\left[y_{i}, y_{i+1}\right]$ as the solutions of the Dirichlet problem

$$
z-z_{x x}=0
$$


with boundary conditions $z\left(t, y_{i}(t)\right)=z_{i}(t), z\left(t, y_{i+1}(t)\right)=$ $z_{i+1}(t)$. The variables $y_{i}$ denote the position of the peaks, and the variables $z_{i}$ denote the values of $z$ at the peaks. In the following part we will show that this property persists for dissipative solutions.

We introduce $\bar{X}=(\bar{y}, \bar{U}, \bar{V}, \bar{M}, \bar{N}, \bar{h})$ as a representative of $z=(u, v)$ in Lagrangian equivalent system; that is, $\bar{X}=$ $L(\bar{z})$, which is given by

$$
\begin{gathered}
\bar{y}(\xi)=\xi \\
\bar{U}(\xi)=\bar{u}(\xi), \quad \bar{V}(\xi)=\bar{v}(\xi), \\
\bar{M}(\xi)=\bar{u}_{x}(\xi), \quad \bar{N}(\xi)=\bar{v}_{x}(\xi), \\
\bar{h}(\xi)=\bar{u}^{2}+\bar{u}_{x}^{2}+\bar{v}^{2}+\bar{v}_{x}^{2} .
\end{gathered}
$$

Let $I=\bigcup_{i=0}^{n} I_{i}$, where $I_{i}$ denote the open interval $\left(\xi_{i}, \xi_{i+1}\right)$ with the conventions that $\xi_{0}=-\infty$ and $\xi_{n+1}=\infty$. For each interval $I_{i}$, we define $\tau_{i}=\inf \left\{\tau(\xi) \mid \xi \in\left(\xi_{i}, \xi_{i+1}\right)\right\}$ such that $\tau_{i}>0$ for all $i=1, \ldots, n$. By the linearity of the governing equations (10), and the bounds which hold on the solution $X$ and $P_{i}, P_{i, x}(i=1,2,3,4)$, it is not hard to check that $y, U, V \in$ $C\left(\left[0, \tau_{i}\right], C^{2}\left(I_{i}\right)\right)$, while $h \in C\left(\left[0, \tau_{i}\right], C^{1}\left(I_{i}\right)\right)$.

Thus the existence of multipeakon solutions is given by the next theorem.

Theorem 3. For any given multipeakon initial data $\bar{z}(x)=(\bar{u}, \bar{v})(x)=\left(\sum_{i=1}^{n} p_{i} e^{-\left|x-\xi_{i}\right|}, \sum_{i=1}^{n} r_{i} e^{-\left|x-\xi_{i}\right|}\right)$, let $(y, U, V, M, N, h)$ be the solution of system (10), (11) with initial data $(\bar{y}, \bar{U}, \bar{V}, \bar{M}, \bar{N}, \bar{H})$ given by (24), (25), and (26). Between adjacent peaks, if $x_{i}=y\left(t, \xi_{i}\right) \neq x_{i+1}=y\left(t, \xi_{i+1}\right)$, the solution $z(t, x)=(u, v)(t, x)$ is twice differentiable with respect to the space variable, and one has

$$
\left(z-z_{x x}\right)(t, x)=0 \quad \text { for } x \in\left(x_{i}, x_{i+1}\right) .
$$

Proof. For a given time $t$, we consider two adjacent peaks $x_{i}=$ $y\left(t, \xi_{i}\right)$ and $x_{i+1}=y\left(t, \xi_{i+1}\right)$. If $x_{i}=x_{i+1}$, then the two peaks have collided and, since $y_{\xi}$ is positive, we must have $y_{\xi}(t, \xi)=$ 0 for all $\xi \in I_{i}$. Hence, $t \geq \tau_{i}$, which conversely implies that $t<$ $\tau_{i}$ when $x_{i}(t)<x_{i+1}(t)$. There exists $\xi \in I_{i}$ such that $x=y(t, \xi)$ for any $x \in\left(x_{i}(t), x_{i+1}(t)\right)$. Since $\xi \in I_{i}$ and $t<\tau_{i}$, we have $y_{\xi}(t, \xi) \neq 0$. It follows from the implicit function theorem that $y(t, \cdot)$ is invertible in a neighborhood of $\xi$ and its inverse is $C^{2}$, and therefore $(u, v)\left(t, x^{\prime}\right)=(U, V)\left(t, y^{-1}\left(t, x^{\prime}\right)\right)$ are $C^{2}$ with respect to the spatial variable and the quantity $\left(z-z_{x x}\right)(t, x)$ is defined in the classical sense.

We now prove that $\left(z-z_{x x}\right)(t, x)=0$ for $x \in\left(x_{i}, x_{i+1}\right)$. Let us first prove that $u-u_{x x}=0$. Assuming that $y_{\xi}(t, \xi) \neq 0$, we have that

$$
\begin{gathered}
u_{x} \circ y=M, \\
u_{x x} \circ y=\frac{M_{\xi}}{y_{\xi}}=\frac{\left(U_{\xi \xi} y_{\xi}-y_{\xi \xi} U_{\xi}\right)}{y_{\xi}^{3}},
\end{gathered}
$$

and therefore

$$
\left(u-u_{x x}\right) \circ y=\frac{\left(U y_{\xi}^{3}-U_{\xi \xi} y_{\xi}+y_{\xi \xi} U_{\xi}\right)}{y_{\xi}^{3}} .
$$

We set

$$
R=U y_{\xi}^{3}-U_{\xi \xi} y_{\xi}+y_{\xi \xi} U_{\xi}
$$

For a given $\xi \in I$ and $t<\tau_{i}$, differentiating (30) with respect to $t$, it then follows from (10) and (11) that

$$
\begin{aligned}
\frac{d R}{d t}= & 3 U y_{\xi}^{2} y_{\xi t}+U_{t} y_{\xi}^{3}-U_{\xi \xi t} y_{\xi}-U_{\xi \xi} y_{\xi t} \\
& +y_{\xi \xi t} U_{\xi}+y_{\xi \xi} U_{\xi t} \\
= & 2 U\left(U_{\xi}+V_{\xi}\right) y_{\xi}^{2}-2 N\left(M_{\xi}-N_{\xi}\right) y_{\xi}^{2} \\
& -\frac{h_{\xi} y_{\xi}}{2}+\frac{h y_{\xi \xi}}{2} .
\end{aligned}
$$

Differentiating (15) with respect to $\xi$, we get

$$
\begin{aligned}
y_{\xi \xi} h+y_{\xi} h_{\xi}= & 2 y_{\xi} y_{\xi \xi} U^{2}+2 y_{\xi}^{2} U U_{\xi}+2 U_{\xi} U_{\xi \xi} \\
& +2 y_{\xi} y_{\xi \xi} V^{2}+2 y_{\xi}^{2} V V_{\xi}+2 V_{\xi} V_{\xi \xi} .
\end{aligned}
$$

We have, after inserting the value of $y_{\xi} h_{\xi}$ given by (32) into (31) and multiplying the equation by $y_{\xi}$, that

$$
\begin{aligned}
y_{\xi} \frac{d R}{d t}= & U U_{\xi} y_{\xi}^{3}-U_{\xi} U_{\xi \xi} y_{\xi} \\
& +\left(h y_{\xi}-y_{\xi}^{2} U^{2}-y_{\xi}^{2} V^{2}-V_{\xi}^{2}\right) y_{\xi \xi} \\
& +U V_{\xi} y_{\xi}^{3}-U_{\xi \xi} V_{\xi} y_{\xi}+U_{\xi} V_{\xi} y_{\xi \xi} .
\end{aligned}
$$

Since $y_{\xi t}=\left(U_{\xi}+V_{\xi}\right)$, it follows from (15) that

$$
y_{\xi} \frac{d R}{d t}=y_{\xi t} \cdot R
$$

For any $\xi \in I$, as $\bar{u}$ is a multipeakon initial data, we have $R(0, \xi)=\left(\bar{u}-\bar{u}_{x x}\right) \cdot y_{\xi}^{3}=0$. It thus follows from Gronwall's lemma that $R(t, \xi)=0$ and therefore $\left(u-u_{x x}\right)(t, \xi)=0$ for $t \in\left[0, \tau_{i}\right]$. Similarly, we can obtain that $\left(v-v_{x x}\right)(t, \xi)=0$.

Thus, the system of ordinary differential equations that the dissipative multipeakon solutions satisfy can be derived based on the fact that the multipeakon structure is preserved by the semigroup of dissipative solutions.

Let us define

$$
H_{i}=\int_{\xi_{i}}^{\xi_{i+1}} h(\xi) d \xi
$$

For each $i=1,2 \ldots, n$, by using (11), we obtain the following system of O.D.E.; namely,

$$
\begin{gathered}
\frac{d y_{i}}{d t}=u_{i}+v_{i}, \quad \frac{d u_{i}}{d t}=-P_{1, i}-P_{2, x i}, \\
\frac{d v_{i}}{d t}=-P_{3, i}-P_{4, x i}, \\
\frac{d H_{i}}{d t}=\left(u_{i+1}^{3}-2 u_{i+1} P_{2, i+1}+v_{i+1}^{3}-2 v_{i+1} P_{4, i+1}\right) \\
-\left(u_{i}^{3}-2 u_{i} P_{2, i}+v_{i}^{3}-2 v_{i} P_{4, i}\right),
\end{gathered}
$$


where $\left(y_{i}, u_{i}, v_{i}\right)=(y, U, V)\left(t, \xi_{i}\right), P_{k, i}=P_{k}\left(t, \xi_{i}\right), P_{k, x i}=$ $P_{k, x}\left(t, \xi_{i}\right),(k=1,2,3,4)$, respectively. We have

$$
\begin{aligned}
& P_{2, i}=\frac{1}{4} \cdot \int_{\left\{t<\tau\left(\xi^{\prime}\right)\right\}} e^{-\left|y_{i}-y\left(\xi^{\prime}\right)\right|} \\
& \times\left[h+\left(U^{2}+2 M N-N^{2}\right) y_{\xi}\right]\left(\xi^{\prime}\right) d \xi^{\prime} .
\end{aligned}
$$

We denote $B=\left\{\xi^{\prime} \in R \mid y_{\xi}\left(t, \xi^{\prime}\right)>0\right\}$. Thus, we get

$$
\begin{gathered}
P_{2, i}=\frac{1}{2} \cdot \int_{B} e^{-\left|y_{i}-y\left(\xi^{\prime}\right)\right|}\left(u^{2}+\frac{u_{x}^{2}}{2}+u_{x} v_{x}+\frac{v^{2}}{2}-\frac{v_{x}^{2}}{2}\right) \\
\circ y\left(\xi^{\prime}\right) y_{\xi}\left(\xi^{\prime}\right) d \xi^{\prime} \\
=\frac{1}{2} \cdot \int_{y(B)} e^{-\left|y_{i}-x\right|}\left(u^{2}+\frac{u_{x}^{2}}{2}+u_{x} v_{x}+\frac{v^{2}}{2}-\frac{v_{x}^{2}}{2}\right) \\
\quad \times(x) d x,
\end{gathered}
$$

where we have used the fact that $h=\left(u^{2}+u_{x}^{2}+v^{2}+v_{x}^{2}\right) \circ y y_{\xi}$ on $B$ and $t<\tau\left(\xi^{\prime}\right)$ if and only if $y_{\xi}\left(t, \xi^{\prime}\right)>0$. Since $y_{\xi}(\xi)=0$ on $B^{c}$, that means $\left(y\left(B^{c}\right)\right)=\int_{y\left(B^{c}\right)} d x=\int_{B^{c}} y_{\xi}(\xi) d \xi=0$, which implies that the domain of integration in (37) can be extended to the whole axis,

$$
P_{2, i}=\frac{1}{2} \cdot \int_{R} e^{-\left|y_{i}-x\right|}\left(u^{2}+\frac{u_{x}^{2}}{2}+u_{x} v_{x}+\frac{v^{2}}{2}-\frac{v_{x}^{2}}{2}\right) d x .
$$

Similarly, we can get that

$$
\begin{gathered}
P_{1, i}=\frac{1}{2} \cdot \int_{R} e^{-\left|y_{i}-x\right|}\left(u v_{x}\right) d x, \\
P_{1, x i}=-\frac{1}{2} \cdot \int_{R} \operatorname{sgn}\left(y_{i}-x\right) e^{-\left|y_{i}-x\right|}\left(u v_{x}\right) d x, \\
P_{2, x i}=-\frac{1}{2} \cdot \int_{R} \operatorname{sgn}\left(y_{i}-x\right) e^{-\left|y_{i}-x\right|} \\
\times\left(u^{2}+\frac{u_{x}^{2}}{2}+u_{x} v_{x}+\frac{v^{2}}{2}-\frac{v_{x}^{2}}{2}\right) d x,
\end{gathered}
$$

$$
\begin{gathered}
P_{3, i}=\frac{1}{2} \cdot \int_{R} e^{-\left|y_{i}-x\right|}\left(v u_{x}\right) d x, \\
P_{3, x i}=-\frac{1}{2} \cdot \int_{R} \operatorname{sgn}\left(y_{i}-x\right) e^{-\left|y_{i}-x\right|}\left(v u_{x}\right) d x, \\
P_{4, i}=\frac{1}{2} \cdot \int_{R} e^{-\left|y_{i}-x\right|} \\
\times\left(v^{2}+\frac{v_{x}^{2}}{2}+u_{x} v_{x}+\frac{u^{2}}{2}-\frac{u_{x}^{2}}{2}\right) d x, \\
P_{4, x i}=-\frac{1}{2} \cdot \int_{R} \operatorname{sgn}\left(y_{i}-x\right) e^{-\left|y_{i}-x\right|} \\
\times\left(v^{2}+\frac{v_{x}^{2}}{2}+u_{x} v_{x}+\frac{u^{2}}{2}-\frac{u_{x}^{2}}{2}\right) d x .
\end{gathered}
$$

Between two adjacent peaks located at $y_{i}$ and $y_{i+1}$, we know that $z=(u, v)$ satisfies $\left(z-z_{x x}\right)=0$ and therefore $z=(u, v)$ can be written as

$$
z(x)=(u, v)(x)=\left(A_{i} e^{x}+B_{i} e^{-x}, C_{i} e^{x}+D_{i} e^{-x}\right)
$$

for $x \in\left[y_{i}, y_{i+1}\right], i=1,2, \ldots, n-1$, where the constants $A_{i}$, $B_{i}, C_{i}$, and $D_{i}$ depend on $u_{i}, u_{i+1}, v_{i}, v_{i+1}, y_{i}$, and $y_{i+1}$ and read

$$
\begin{aligned}
& A_{i}=\frac{e^{-\bar{y}_{i}}}{2}\left[\frac{\bar{u}_{i}}{\cosh \left(\delta y_{i}\right)}+\frac{\delta u_{i}}{\sinh \left(\delta y_{i}\right)}\right], \\
& B_{i}=\frac{e^{\bar{y}_{i}}}{2}\left[\frac{\bar{u}_{i}}{\cosh \left(\delta y_{i}\right)}-\frac{\delta u_{i}}{\sinh \left(\delta y_{i}\right)}\right], \\
& C_{i}=\frac{e^{-\bar{y}_{i}}}{2}\left[\frac{\bar{v}_{i}}{\cosh \left(\delta y_{i}\right)}+\frac{\delta v_{i}}{\sinh \left(\delta y_{i}\right)}\right], \\
& D_{i}=\frac{e^{\bar{y}_{i}}}{2}\left[\frac{\bar{v}_{i}}{\cosh \left(\delta y_{i}\right)}-\frac{\delta v_{i}}{\sinh \left(\delta y_{i}\right)}\right],
\end{aligned}
$$

where

$$
\begin{array}{ll}
\bar{y}_{i}=\frac{1}{2} \cdot\left(y_{i}+y_{i+1}\right), & \delta y_{i}=\frac{1}{2} \cdot\left(y_{i}-y_{i+1}\right), \\
\bar{u}_{i}=\frac{1}{2} \cdot\left(u_{i}+u_{i+1}\right), & \delta u_{i}=\frac{1}{2} \cdot\left(u_{i}-u_{i+1}\right), \\
\bar{v}_{i}=\frac{1}{2} \cdot\left(v_{i}+v_{i+1}\right), & \delta v_{i}=\frac{1}{2} \cdot\left(v_{i}-v_{i+1}\right) .
\end{array}
$$

Thus, the constants $A_{i}, B_{i}, C_{i}$, and $D_{i}$ uniquely determine $z=$ $(u, v)$ on the interval $\left[y_{i}, y_{i+1}\right]$, and we compute

$$
\begin{aligned}
\delta H_{i}= & H_{i+1}-H_{i}=\int_{y_{i}}^{y_{i+1}}\left(u^{2}+u_{x}^{2}+v^{2}+v_{x}^{2}\right) d x \\
= & 2 \bar{u}_{i}^{2} \tanh \left(\delta y_{i}\right)+2 \delta u_{i}^{2} \operatorname{coth}\left(\delta y_{i}\right) \\
& +2 \bar{v}_{i}^{2} \tanh \left(\delta y_{i}\right)+2 \delta v_{i}^{2} \operatorname{coth}\left(\delta y_{i}\right) \\
= & \delta H_{1 i}+\delta H_{2 i},
\end{aligned}
$$


with $\delta H_{1 i}=2 \bar{u}_{i}^{2} \tanh \left(\delta y_{i}\right)+2 \delta u_{i}^{2} \cosh \left(\delta y_{i}\right)$ and $\delta H_{2 i}=$ $2 \bar{v}_{i}^{2} \tanh \left(\delta y_{i}\right)+2 \delta v_{i}^{2} \cosh \left(\delta y_{i}\right)$. We now turn to the computation of $P_{k, i}(k=1,2,3,4)$ given by (39) and $(40)$. Let us write $z=(u, v)$ as

$$
\begin{aligned}
z(t, x)= & (u, v)(t, x) \\
= & \left(\sum_{j=0}^{n}\left(A_{j} e^{x}+B_{j} e^{-x}\right) \chi_{\left(y_{j}, y_{j+1}\right)}(x),\right. \\
& \left.\sum_{j=0}^{n}\left(C_{j} e^{x}+D_{j} e^{-x}\right) \chi_{\left(y_{j}, y_{j+1}\right)}(x)\right) .
\end{aligned}
$$

Set $y_{0}=-\infty, y_{n+1}=\infty, u_{0}=u_{n+1}=0, v_{0}=v_{n+1}=0$, $A_{0}=u_{1} e^{-y_{1}}, B_{0}=0, A_{n}=0, B_{n}=u_{n} e^{y_{n}}, C_{0}=v_{1} e^{-y_{1}}$, $D_{0}=0, C_{n}=0$, and $D_{n}=v_{n} e^{y_{n}}$. We have

$$
\begin{gathered}
u v_{x}=\sum_{j=0}^{n}\left(A_{j} C_{j} e^{2 x}-A_{j} D_{j}+B_{j} C_{j}\right. \\
\left.-B_{j} D_{j} e^{-2 x}\right) \chi_{\left(y_{j}, y_{j+1}\right)} \\
u^{2}+\frac{u_{x}^{2}}{2}+u_{x} v_{x}+\frac{v^{2}}{2}-\frac{v_{x}^{2}}{2} \\
=\sum_{j=0}^{n}\left(\left(\frac{3}{2} \cdot A_{j}^{2}+A_{j} C_{j}\right) e^{2 x}\right. \\
+\left(A_{j} B_{j}-A_{j} D_{j}-B_{j} C_{j}+2 C_{j} D_{j}\right) \\
\left.+\left(\frac{3}{2} \cdot B_{j}^{2}+B_{j} D_{j}\right) e^{-2 x}\right) \chi_{\left(y_{j}, y_{j+1}\right)}, \\
v u_{x}=\sum_{j=0}^{n}\left(A_{j} C_{j} e^{2 x}-C_{j} B_{j}+D_{j} A_{j}\right. \\
\left.+B_{j} D_{j} e^{-2 x}\right) \chi_{\left(y_{j}, y_{j+1}\right)} \\
\left.+\left(\frac{3}{2} \cdot D_{j}^{2}+D_{j} B_{j}\right) e^{-2 x}\right) \chi_{\left(y_{j}, y_{j+1}\right)} . \\
v^{2}+\frac{v_{x}^{2}}{2}+u_{x} v_{x}+\frac{u^{2}}{2}-\frac{u_{x}^{2}}{2} \\
=\sum_{j=0}^{n}\left(\left(\frac{3}{2} \cdot C_{j}^{2}+A_{j} C_{j}\right) e^{2 x}\right. \\
+\left(C_{j} D_{j}-C_{j} B_{j}-D_{j} A_{j}+2 A_{j} B_{j}\right)
\end{gathered}
$$

By inserting (46) into (39) and (40), we get

$$
\begin{gathered}
P_{1, i}=\frac{1}{2} \cdot \sum_{j=0}^{n} \int_{y_{j}}^{y_{j+1}} e^{-k_{i j}\left(y_{i}-x\right)} \\
\times\left(A_{j} C_{j} e^{2 x}-A_{j} D_{j}+B_{j} C_{j}-B_{j} D_{j} e^{-2 x}\right) d x, \\
P_{2, i}=\frac{1}{2} \sum_{j=0}^{n} \int_{y_{j}}^{y_{j+1}} e^{-k_{i j}\left(y_{i}-x\right)} \\
\times\left(\left(\frac{3}{2} A_{j}^{2}+A_{j} C_{j}\right) e^{2 x}\right. \\
+\left(A_{j} B_{j}-A_{j} D_{j}-B_{j} C_{j}+2 C_{j} D_{j}\right) \\
\left.\quad+\left(\frac{3}{2} B_{j}^{2}+B_{j} D_{j}\right) e^{-2 x}\right) d x, \\
P_{3, i}=\frac{1}{2} \cdot \sum_{j=0}^{n} \int_{y_{j}}^{y_{j+1}} e^{-k_{i j}\left(y_{i}-x\right)} \\
\times\left(A_{j} C_{j} e^{2 x}-C_{j} B_{j}+D_{j} A_{j}-B_{j} D_{j} e^{-2 x}\right) d x, \\
P_{4, i}=\frac{1}{2} \sum_{j=0}^{n} \int_{y_{j}}^{y_{j+1}} e^{-k_{i j}\left(y_{i}-x\right)} \\
\times\left(\left(\frac{3}{2} C_{j}^{2}+A_{j} C_{j}\right) e^{2 x}\right. \\
\quad+\left(C_{j} D_{j}-C_{j} B_{j}-D_{j} A_{j}+2 A_{j} B_{j}\right) \\
\left.\quad+\left(\frac{3}{2} D_{j}^{2}+D_{j} B_{j}\right) e^{-2 x}\right) d x,
\end{gathered}
$$

where $k_{i j}=-1$ if $i \leq j, k_{i j}=1$ if $i>j$. It then follows from (42) and (44) that

$$
\begin{gathered}
A_{j}^{2}=\frac{e^{-2 \bar{y}_{j}}}{\sinh ^{2}\left(2 \delta y_{j}\right)}\left[\bar{u}_{j}^{2} \sinh ^{2}\left(\delta y_{j}\right)+2 \bar{u}_{j} \delta u_{j} \sinh \left(\delta y_{j}\right)\right. \\
\left.\quad \times \cosh \left(\delta y_{j}\right)+\delta u_{j}^{2} \cosh ^{2}\left(\delta y_{j}\right)\right] \\
=\frac{e^{-2 \bar{y}_{j}}}{4 \sinh \left(2 \delta y_{j}\right)} \cdot\left[\delta H_{1 j}+4 \bar{u}_{j} \delta u_{j}\right], \\
A_{j} B_{j}=\frac{1}{4 \sinh \left(2 \delta y_{j}\right)} \cdot\left[4 \bar{u}_{j}^{2} \tanh \left(\delta y_{j}\right)-\delta H_{1 j}\right], \\
A_{j} C_{j}=\frac{e^{-2 \bar{y}_{j}}}{2 \sinh \left(2 \delta y_{j}\right)}\left[\bar{u}_{j} \bar{v}_{j} \tanh \left(\delta y_{j}\right)+\delta u_{j} \bar{v}_{j}+\delta v_{j} \bar{u}_{j}\right. \\
\left.+\delta u_{j} \delta v_{j} \operatorname{coth}\left(\delta y_{j}\right)\right],
\end{gathered}
$$




$$
\begin{gathered}
A_{j} D_{j}=\frac{1}{2 \sinh \left(2 \delta y_{j}\right)}\left[\bar{u}_{j} \bar{v}_{j} \tanh \left(\delta y_{j}\right)+\delta u_{j} \bar{v}_{j}-\delta v_{j} \bar{u}_{j}\right. \\
\left.-\delta u_{j} \delta v_{j} \operatorname{coth}\left(\delta y_{j}\right)\right], \\
B_{j} C_{j}=\frac{1}{2 \sinh \left(2 \delta y_{j}\right)}\left[\bar{u}_{j} \bar{v}_{j} \tanh \left(\delta y_{j}\right)+\delta v_{j} \bar{u}_{j}-\delta u_{j} \bar{v}_{j}\right. \\
\left.-\delta u_{j} \delta v_{j} \operatorname{coth}\left(\delta y_{j}\right)\right], \\
B_{j} D_{j}=\frac{e^{2 \bar{y}_{j}}}{2 \sinh \left(2 \delta y_{j}\right)}\left[\bar{u}_{j} \bar{v}_{j} \tanh \left(\delta y_{j}\right)-\delta u_{j} \bar{v}_{j}-\delta v_{j} \bar{u}_{j}\right. \\
\left.+\delta u_{j} \delta v_{j} \operatorname{coth}\left(\delta y_{j}\right)\right], \\
B_{j}^{2}=\frac{e^{2 \bar{y}_{j}}}{4 \sinh \left(2 \delta y_{j}\right)} \cdot\left[\delta H_{1 j}-4 \bar{u}_{j} \delta u_{j}\right] .
\end{gathered}
$$

Thus, from (48), we can obtain that

$$
\begin{aligned}
& \int_{y_{j}}^{y_{j+1}} e^{-k_{i j}\left(y_{i}-x\right)} A_{j}^{2} e^{2 x} d x \\
&= \frac{e^{-k_{i j} y_{i}} \cdot e^{k_{i j} \bar{y}_{j}}}{2\left(2+k_{i j}\right) \sinh \left(2 \delta y_{j}\right)} \\
& \times \sinh \left(\left(2+k_{i j}\right) \delta y_{j}\right)\left[\delta H_{1 j}+4 \bar{u}_{j} \delta u_{j}\right] \\
& \int_{y_{j}}^{y_{j+1}} e^{-k_{i j}\left(y_{i}-x\right)} A_{j} C_{j} e^{2 x} d x \frac{e^{-k_{i j} y_{i}} \cdot e^{k_{i j} \bar{y}_{j}}}{\left(2+k_{i j}\right) \sinh \left(2 \delta y_{j}\right)} \\
& \times \sinh \left(\left(2+k_{i j}\right) \delta y_{j}\right) \\
& \cdot\left[\bar{u}_{j} \bar{v}_{j} \tanh \left(\delta y_{j}\right)+\delta u_{j} \bar{v}_{j}+\delta v_{j} \bar{u}_{j}\right. \\
&\left.+\delta u_{j} \delta v_{j} \operatorname{coth}\left(\delta y_{j}\right)\right] \\
& \int_{y_{j}}^{y_{j+1}} e^{-k_{i j}\left(y_{i}-x\right)} A_{j} B_{j} d x \\
& \quad
\end{aligned}
$$

$$
\begin{gathered}
\int_{y_{j}}^{y_{j+1}} e^{-k_{i j}\left(y_{i}-x\right)} A_{j} D_{j} d x \\
=\frac{e^{-k_{i j} y_{i}} \cdot e^{k_{i j} \bar{y}_{j}}}{\sinh \left(2 \delta y_{j}\right)} \sinh \left(\delta y_{j}\right) \\
\cdot\left[\bar{u}_{j} \bar{v}_{j} \tanh \left(\delta y_{j}\right)+\delta u_{j} \bar{v}_{j}-\delta v_{j} \bar{u}_{j}\right. \\
\left.-\delta u_{j} \delta v_{j} \operatorname{coth}\left(\delta y_{j}\right)\right], \\
\int_{y_{j}}^{y_{j+1}} e^{-k_{i j}\left(y_{i}-x\right)} B_{j} C_{j} d x \\
=\frac{e^{-k_{i j} y_{i}} \cdot e^{k_{i j} \bar{y}_{j}}}{\sinh \left(2 \delta y_{j}\right)} \sinh \left(\delta y_{j}\right) \\
\cdot\left[\bar{u}_{j} \bar{v}_{j} \tanh \left(\delta y_{j}\right)+\delta v_{j} \bar{u}_{j}-\delta u_{j} \bar{v}_{j}\right. \\
\left.-\delta u_{j} \delta v_{j} \operatorname{coth}\left(\delta y_{j}\right)\right],
\end{gathered}
$$$$
\int_{y_{j}}^{y_{j+1}} e^{-k_{i j}\left(y_{i}-x\right)} C_{j} D_{j} d x
$$$$
=\frac{e^{-k_{i j} y_{i}} \cdot e^{k_{i j} \bar{y}_{j}}}{2 \sinh \left(2 \delta y_{j}\right)} \sinh \left(\delta y_{j}\right)
$$$$
\times\left[4 \bar{v}_{j}^{2} \tanh \left(\delta y_{j}\right)-\delta H_{2 j}\right],
$$$$
\int_{y_{j}}^{y_{j+1}} e^{-k_{i j}\left(y_{i}-x\right)} B_{j}^{2} e^{-2 x} d x
$$$$
=\frac{e^{-k_{i j} y_{i}} \cdot e^{k_{i j} \bar{y}_{j}}}{2\left(k_{i j}-2\right) \sinh \left(2 \delta y_{j}\right)}
$$$$
\times \sinh \left(\left(k_{i j}-2\right) \delta y_{j}\right)\left[\delta H_{1 j}-4 \bar{u}_{j} \delta u_{j}\right],
$$$$
\int_{y_{j}}^{y_{j+1}} e^{-k_{i j}\left(y_{i}-x\right)} B_{j} D_{j} e^{-2 x} d x
$$$$
=\frac{e^{-k_{i j} y_{i}} \cdot e^{k_{i j} \bar{y}_{j}}}{\left(k_{i j}-2\right) \sinh \left(2 \delta y_{j}\right)}
$$$$
\times \sinh \left(\left(k_{i j}-2\right) \delta y_{j}\right)
$$$$
\cdot\left[\bar{u}_{j} \bar{v}_{j} \tanh \left(\delta y_{j}\right)-\delta u_{j} \bar{v}_{j}-\delta v_{j} \bar{u}_{j}\right.
$$$$
\left.+\delta u_{j} \delta v_{j} \operatorname{coth}\left(\delta y_{j}\right)\right] \text {. }
$$

It thus follows from (49) that

$$
P_{1, i}=\sum_{j=0}^{n} P_{1, i j}, \quad P_{2, i}=\sum_{j=0}^{n} P_{2, i j},
$$


where

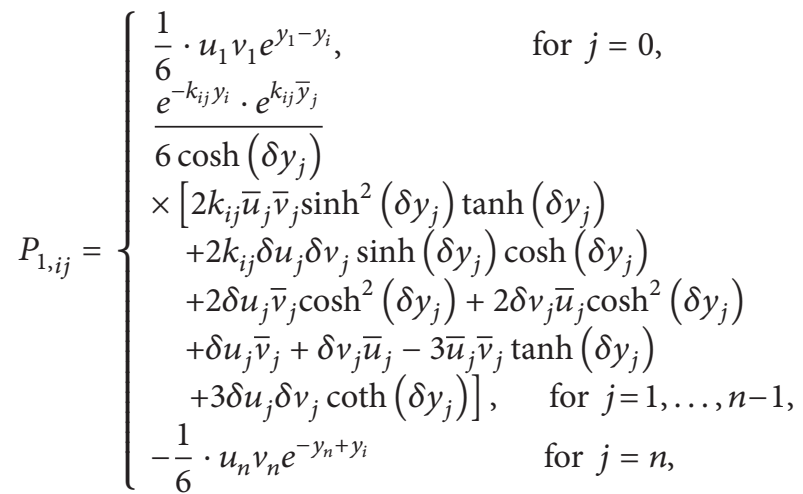

$$
\begin{aligned}
& P_{2, i j}=\left\{\begin{array}{l}
\frac{1}{4} \cdot u_{1}^{2} e^{y_{1}-y_{i}}+\frac{1}{6} \cdot u_{1} v_{1} e^{y_{1}-y_{i}}, \quad \text { for } j=0, \\
\frac{e^{-k_{i j} y_{i}} \cdot e^{k_{i j} \bar{y}_{j}}}{4 \cosh \left(\delta y_{j}\right)} \\
\times \quad\left[H_{1 j} \cosh ^{2}\left(\delta y_{j}\right)+4 k_{i j} \bar{u}_{j} \delta u_{j} \sinh ^{2}\left(\delta y_{j}\right)\right. \\
\quad+2 \bar{u}_{j}^{2} \tanh \left(\delta y_{j}\right)+4 \bar{v}_{j}^{2} \tanh \left(\delta y_{j}\right) \\
\quad-\delta H_{2 j}-\frac{4}{3} \bar{u}_{j} \bar{v}_{j} \tanh \left(\delta y_{j}\right)+\frac{8}{3} \delta u_{j} \delta v_{j} \\
\quad \times \operatorname{coth}\left(\delta y_{j}\right)+\frac{4}{3} \bar{u}_{j} \bar{v}_{j} \cosh \left(\delta y_{j}\right) \sinh \left(\delta y_{j}\right) \\
\quad+\frac{4}{3} \delta u_{j} \delta v_{j} \cdot \operatorname{coth}\left(\delta y_{j}\right) \cosh { }^{2}\left(\delta y_{j}\right) \\
\quad+\frac{4}{3} k_{i j} \delta u_{j} \bar{v}_{j} \sinh ^{2}\left(\delta y_{j}\right)+\frac{4}{3} \\
\left.\quad \times k_{i j} \delta v_{j} \bar{u}_{j} \sinh ^{2}\left(\delta y_{j}\right)\right], \quad \text { for } j=1, \ldots, n-1, \\
\frac{1}{4} \cdot u_{n}^{2} e^{-y_{n}+y_{i}}+\frac{1}{6} \cdot u_{n} v_{n} e^{-y_{n}+y_{i}}, \quad \text { for } j=n .
\end{array}\right.
\end{aligned}
$$

The terms $P_{3, i}, P_{4, i}$, and $P_{k, i x}(k=1,2,3,4)$ can be computed in the same way and we have

$$
P_{k, i x}=-\sum_{j=0}^{n} k_{i j} P_{k, i j} \cdot(k=1,2,3,4) .
$$

The result can be summarized in the following theorem.

Theorem 4. Assume $\bar{y}_{i}=\xi_{i}, \bar{z}_{i}=\left(\bar{u}_{i}, \bar{v}_{i}\right)=\left(\bar{u}\left(\xi_{i}\right), \bar{v}\left(\xi_{i}\right)\right)$ and $\bar{H}_{i}=\int_{\xi_{i}}^{\xi_{i+1}}\left(\bar{u}^{2}+\bar{u}_{x}^{2}+\bar{v}^{2}+\bar{v}_{x}^{2}\right) d x$ for $i=1, \ldots, n$ with a multipeakon initial data $\bar{z}=(\bar{u}, \bar{v})$ as given by (22). Then, there exists a global solution $\left(y_{i}, u_{i}, v_{i}, H_{i}\right)$ of (36), (50), and (52) with initial data $\left(\bar{y}_{i}, \bar{u}_{i}, \bar{v}_{i}, \bar{H}_{i}\right)$. On each interval $\left[y_{i}(t), y_{i+1}(t)\right]$, one defines $z(t, x)=(u, v)(t, x)$ as the solution of the Dirichlet problem $z-z_{x x}=0$ with boundary conditions $z\left(t, y_{i}(t)\right)=z_{i}(t), z\left(t, y_{i+1}(t)\right)=z_{i+1}(t)$ for each time $t$. Thus $z=(u, v)$ is a dissipative solution of the modified coupled two-component Camassa-Holm system, which is the dissipative multipeakon solution.

\section{Examples}

In this section, we give the examples with the case $n=1$ by explicit calculations and the case $n=2$ by numerical computations with peakon-antipeakon collisions. (i) Let $n=1$. From (50) and (52), we can compute that $P_{1,1}=\sum_{j=0,1} P_{1,1 j}=u_{1} v_{1} / 6-u_{1} v_{1} / 6=0$ and $P_{2, x 1}=-k_{1 j} \sum_{j=0,1} P_{2,1 j}=-u_{1}^{2} / 4-u_{1} v_{1} / 6+u_{1}^{2} / 4+$ $u_{1} v_{1} / 6=0$, which imply that $u_{1 t}=-P_{1,1}-P_{2, x 1}=$ 0 and therefore $u_{1}=c_{1}$. Similarly, we can get that $v_{1}=c_{2}$. Thus from (36), we can obtain that $y_{1 t}=$ $u_{1}+v_{1}=c_{1}+c_{2}=c$, which yields $y_{1}=c t+a$ with $c_{1}, c_{2}, c, a$ some constants. There is no collision and we find the familiar one peakon $(u, v)(t, x)=$ $\left(c_{1} e^{-\left|x-c_{1} t-a\right|}, c_{2} e^{-\left|x-c_{2} t-a\right|}\right)$.

(ii) Let $n=2$. We first consider the case of an antisymmetric pair of peakons where the two peakons collide. We take the initial conditions as

$$
\begin{gathered}
y_{2}(0)=-y_{1}(0)=\bar{y}, \quad u_{2}(0)=-u_{1}(0)=\bar{u}, \\
v_{2}(0)=-v_{1}(0)=\bar{v}, \quad \delta H_{1}(0)=E^{2}
\end{gathered}
$$

for some strictly positive constants $\bar{y}, \bar{u}, \bar{v}$, and $E$ the initial total energy of the system, that is, the $H^{1}$ norm of the solution; we denote $\tau=\tau_{1}$, the time of collision. For $t<\tau$, the solution is identical to the conservative case. After collision, for $t \geq \tau$, the solution remains antisymmetric. Let us assume this for the moment and write

$$
\begin{array}{cl}
y=y_{2}=-y_{1}, & u=u_{2}=-u_{1}, \\
v=v_{2}=-v_{1}, & h=\delta H_{1}, \\
P_{2}=P_{2,1}=P_{2,2}, & P_{4}=P_{4,1}=P_{4,2}, \\
P_{1}=P_{1,1}=-P_{1,2}, & P_{3}=P_{3,1}=-P_{3,2}, \\
P_{2, x}=P_{2, x 1}=-P_{2, x 2}, & P_{4, x}=P_{4, x 1}=-P_{4, x 2} .
\end{array}
$$

By using (50) and (52) and after some calculations, we can compute $P_{k}$ and $P_{k, x}(k=1,2,3,4)$ and obtain that

$$
\begin{gathered}
P_{1}=\sum_{j=0,2} P_{1,1 j}=\frac{1}{6} \cdot u v\left(1-e^{-2 y}\right), \\
P_{2}=\sum_{j=0,2} P_{2,1 j}=\left(\frac{1}{4} \cdot u^{2}+\frac{1}{6} \cdot u v\right)\left(1+e^{-2 y}\right), \\
P_{3}=\sum_{j=0,2} P_{3,1 j}=\frac{1}{6} \cdot u v\left(1-e^{-2 y}\right), \\
P_{4}=\sum_{j=0,2} P_{4,1 j}=\left(\frac{1}{4} \cdot v^{2}+\frac{1}{6} \cdot u v\right)\left(1+e^{-2 y}\right), \\
P_{2, x}=-\left(-\sum_{j=0,2} k_{1, j} P_{2,1 j}\right) \\
=-\left(\frac{1}{4} \cdot u^{2}+\frac{1}{6} \cdot u v\right)\left(1-e^{-2 y}\right), \\
P_{4, x}=-\left(-\sum_{j=0,2} k_{1, j} P_{4,1 j}\right) \\
=-\left(\frac{1}{4} \cdot v^{2}+\frac{1}{6} \cdot u v\right)\left(1-e^{-2 y}\right) .
\end{gathered}
$$


Thus we are led to the following system of ordinary differential equations:

$$
\begin{gathered}
y_{t}=u+v, \quad u_{t}=\frac{1}{4} \cdot u^{2}\left(1-e^{-2 y}\right), \\
v_{t}=\frac{1}{4} \cdot v^{2}\left(1-e^{-2 y}\right) \\
h_{t}=\left(u^{3}+v^{3}\right)\left(1-e^{-2 y}\right)-\frac{2}{3} \cdot u v(u+v)\left(1+e^{-2 y}\right) .
\end{gathered}
$$

Note that this system holds before collision. With the initial condition $y(\tau)=u(\tau)=v(\tau)=0$, the solution of (56) is $y(t)=u(t)=v(t)=0$ and $h(t)=h(\tau)$. It means that the multipeakon solution remains identically equal to zero after the collision.

If we consider a more general case with two colliding peakons by using the Hamiltonian system before collision, then from (50) and (52), the system (36) can be rewritten as

$$
\begin{gathered}
\frac{d y_{1}}{d t}=u_{1}+v_{1}, \quad \frac{d y_{2}}{d t}=u_{2}+v_{2}, \\
\frac{d u_{1}}{d t}=\frac{1}{4} \cdot u_{1}^{2}-\frac{1}{4} \cdot u_{2}^{2} e^{y_{1}-y_{2}}, \\
\frac{d u_{2}}{d t}=\frac{1}{4} \cdot u_{1}^{2} e^{y_{1}-y_{2}}-\frac{1}{4} \cdot u_{2}^{2}, \\
\frac{d v_{1}}{d t}=\frac{1}{4} \cdot v_{1}^{2}-\frac{1}{4} \cdot v_{2}^{2} e^{y_{1}-y_{2}}, \\
\frac{d v_{2}}{d t}=\frac{1}{4} \cdot v_{1}^{2} e^{y_{1}-y_{2}}-\frac{1}{4} \cdot v_{2}^{2}, \\
\frac{d H_{1}}{d t}=\left(u_{2}^{3}-2 u_{2} P_{2,2}+v_{2}^{3}-2 v_{2} P_{4,2}\right) \\
-\left(u_{1}^{3}-2 u_{1} P_{2,1}+v_{1}^{3}-2 v_{1} P_{4,1}\right) .
\end{gathered}
$$

Thus we have

$$
\begin{aligned}
& y_{1}=\ln \left(\frac{c_{1}-c_{2}}{c_{1} e^{-c_{1}(t-\tau)}-c_{2} e^{-c_{2}(t-\tau)}}\right), \\
& y_{2}=\ln \left(\frac{c_{1} e^{c_{1}(t-\tau)}-c_{2} e^{c_{2}(t-\tau)}}{c_{1}-c_{2}}\right), \\
& u_{1}=\frac{\left(c_{1}^{1}\right)^{2}-\left(c_{2}^{1}\right)^{2} e^{\left(c_{1}^{1}-c_{2}^{1}\right)(t-\tau)}}{c_{1}^{1}-c_{2}^{1} e^{\left(c_{1}^{1}-c_{2}^{1}\right)(t-\tau)}}, \\
& u_{2}=\frac{\left(c_{2}^{1}\right)^{2}-\left(c_{1}^{1}\right)^{2} e^{\left(c_{1}^{1}-c_{2}^{1}\right)(t-\tau)}}{c_{2}^{1}-c_{1}^{1} e^{\left(c_{1}^{1}-c_{2}^{1}\right)(t-\tau)}}, \\
& v_{1}=\frac{\left(c_{1}^{2}\right)^{2}-\left(c_{2}^{2}\right)^{2} e^{\left(c_{1}^{2}-c_{2}^{2}\right)(t-\tau)}}{c_{1}^{2}-c_{2}^{2} e^{\left(c_{1}^{2}-c_{2}^{2}\right)(t-\tau)}}, \\
& v_{2}=\frac{\left(c_{2}^{2}\right)^{2}-\left(c_{1}^{2}\right)^{2} e^{\left(c_{1}^{2}-c_{2}^{2}\right)(t-\tau)}}{c_{2}^{2}-c_{1}^{2} e^{\left(c_{1}^{2}-c_{2}^{2}\right)(t-\tau)}},
\end{aligned}
$$

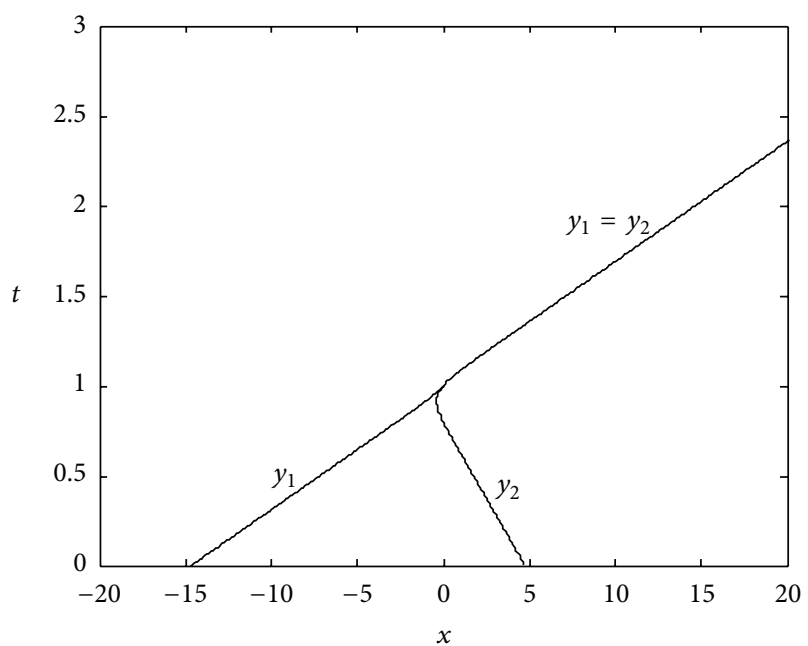

FIgURe 1: Position of the peakons.

where $c_{1}=c_{1}^{1}+c_{1}^{2}$ and $c_{2}=c_{2}^{1}+c_{2}^{2}$ denote the speed of the peaks $y_{1}$ and $y_{2}$, respectively. At collision time, we have

$$
\begin{gathered}
y_{1}(\tau)=y_{2}(\tau)=0, \\
u_{1}(\tau)=u_{2}(\tau)=c_{1}^{1}+c_{2}^{1}, \\
v_{1}(\tau)=v_{2}(\tau)=c_{1}^{2}+c_{2}^{2} .
\end{gathered}
$$

For the initial data given by (59), the solution of (57) is

$$
\begin{array}{cc}
u_{1}(t)=u_{2}(t)=c_{1}^{1}+c_{2}^{1}, \quad v_{1}(t)=v_{2}(t)=c_{1}^{2}+c_{2}^{2}, \\
y_{1}=y_{2}=\left(c_{1}+c_{2}\right) t, \quad H_{1}(t)=H_{1}(\tau),
\end{array}
$$

with $c_{1}=c_{1}^{1}+c_{1}^{2}, c_{2}=c_{2}^{1}+c_{2}^{2}$, and after the collision we obtain a single peakon traveling at speed $c_{1}+c_{2}$.

We know that if $p_{i}(0)$ and $r_{i}(0)$ have the same sign for all $i=1,2, \ldots, n$ and $q_{i}(t)$ remain distinct, (57) admits a unique global solution, where the peakons are traveling in the same direction. However, when two peakons have opposite signs, collisions may occur, and if so, the system (57) blows up. In Figures 1 and 2, the solution is plotted with $c_{1}=15, c_{2}=-5$, and $\tau=1$.

\section{Conclusion}

Considered in this paper is the dissipative property of the modified coupled two-component Camassa-Holm system after wave breaking. Based on the obtained global dissipative solutions of the modified coupled two-component CamassaHolm system, we construct the dissipative multipeakon solutions, a useful result for understanding the inevitable multipeakon phenomenon near wave breaking. Some interesting issues worthy of further investigation include the computational complexity of the algorithms and the impact of the multipeakon dissipative behavior on the system performance when blended into control system design. 


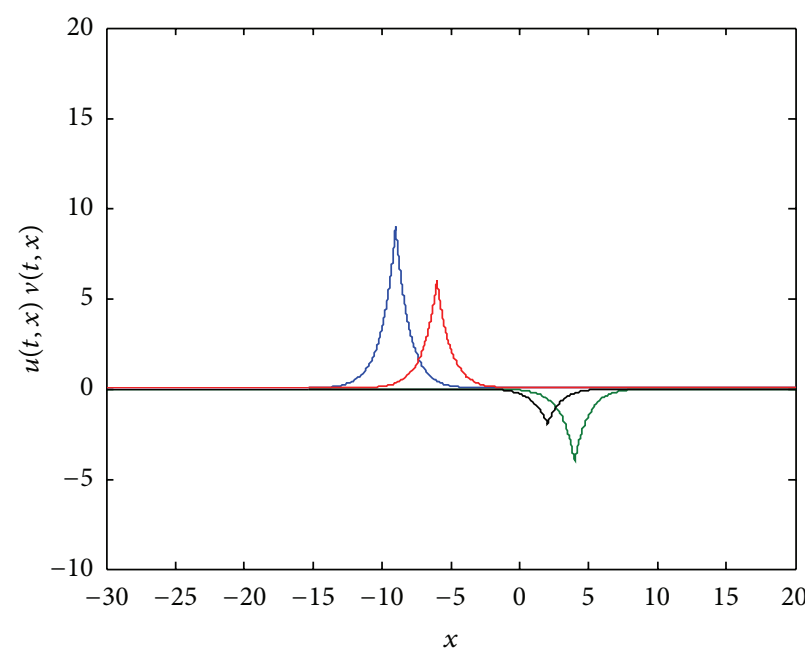

(a)

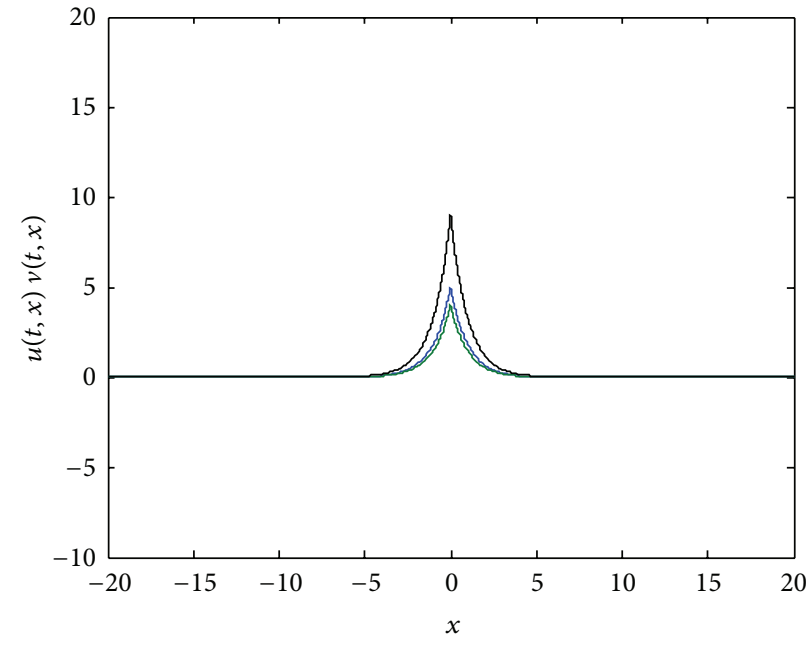

(b)

FIgURE 2: (a) Initial configuration. (b) The two peakons collide.

\section{Acknowledgments}

The paper is supported by the Major State Basic Research Development Program 973 (no. 2012CB215202), the National Natural Science Foundation of China (no. 61134001), and the Fundamental Research Funds for the Central Universities (no. CDJXS12170003). The authors would like to thank the referees.

\section{References}

[1] G. A. Nariboli, "Nonlinear longitudinal dispersive waves in elastic rods," Journal of Mathematical Physics, vol. 4, pp. 64-73, 1970.

[2] G. B. Whitham, Linear and Nonlinear Waves, John Wiley \& Sons, New York, NY, USA, 1980.

[3] A. Constantin, "The Hamiltonian structure of the CamassaHolm equation," Expositiones Mathematicae, vol. 15, no. 1, pp. 53-85, 1997.

[4] R. Camassa and D. D. Holm, "An integrable shallow water equation with peaked solitons," Physical Review Letters, vol. 71, no. 11, pp. 1661-1664, 1993.

[5] H. R. Dullin, G. A. Gottwald, and D. D. Holm, "An integrable shallow water equation with linear and nonlinear dispersion," Physical Review Letters, vol. 87, no. 19, Article ID 194501, 4 pages, 2001.

[6] A. Constantin and D. Lannes, "The hydrodynamical relevance of the Camassa-Holm and Degasperis-Procesi equations," Archive for Rational Mechanics and Analysis, vol. 192, no. 1, pp. 165-186, 2009.

[7] R. S. Johnson, "The Camassa-Holm equation for water waves moving over a shear flow," Japan Society of Fluid Mechanics: Fluid Dynamics Research, vol. 33, no. 1-2, pp. 97-111, 2003.

[8] A. Constantin and J. Escher, "Wave breaking for nonlinear nonlocal shallow water equations," Acta Mathematica, vol. 181, no. 2, pp. 229-243, 1998.

[9] A. Constantin, "Existence of permanent and breaking waves for a shallow water equation: a geometric approach," Annales de l'Institut Fourier, vol. 50, no. 2, pp. 321-362, 2000.
[10] A. Bressan and A. Constantin, "Global conservative solutions of the Camassa-Holm equation," Archive for Rational Mechanics and Analysis, vol. 183, no. 2, pp. 215-239, 2007.

[11] H. Holden and X. Raynaud, "Global conservative solutions of the Camassa-Holm equation-a Lagrangian point of view," Communications in Partial Differential Equations, vol. 32, no. 10-12, pp. 1511-1549, 2007.

[12] H. Holden and X. Raynaud, "Periodic conservative solutions of the Camassa-Holm equation," Annales de l'Institut Fourier, vol. 58, no. 3, pp. 945-988, 2008.

[13] A. Bressan and A. Constantin, "Global dissipative solutions of the Camassa-Holm equation," Analysis and Applications, vol. 5, no. 1, pp. 1-27, 2007.

[14] H. Holden and X. Raynaud, "Dissipative solutions for the Camassa-Holm equation," Discrete and Continuous Dynamical Systems A, vol. 24, no. 4, pp. 1047-1112, 2009.

[15] H. Holden and X. Raynaud, "Global conservative multipeakon solutions of the Camassa-Holm equation," Journal of Hyperbolic Differential Equations, vol. 4, no. 1, pp. 39-64, 2007.

[16] H. Holden and X. Raynaud, "Global dissipative multipeakon solutions of the Camassa-Holm equation," Communications in Partial Differential Equations, vol. 33, no. 10-12, pp. 2040-2063, 2008.

[17] Y. Fu, Y. Liu, and C. Qu, "Well-posedness and blow-up solution for a modified two-component periodic Camassa-Holm system with peakons," Mathematische Annalen, vol. 348, no. 2, pp. 415$448,2010$.

[18] Y. Fu and C. Qu, "Well posedness and blow-up solution for a new coupled Camassa-Holm equations with peakons," Journal of Mathematical Physics, vol. 50, no. 1, Article ID 012906, 25 pages, 2009.

[19] L. Tian, Y. Wang, and J. Zhou, "Global conservative and dissipative solutions of a coupled Camassa-Holm equations," Journal of Mathematical Physics, vol. 52, no. 6, Article ID 063702, 29 pages, 2011.

[20] Y. Wang and Y. Song, "On the global existence of dissipative solutions for the modified coupled Camassa-Holm system," Soft Computing. In press. 


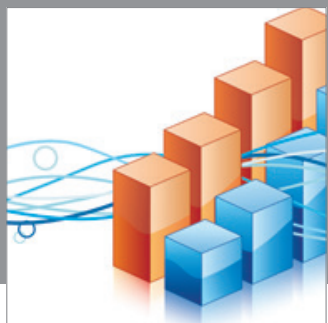

Advances in

Operations Research

mansans

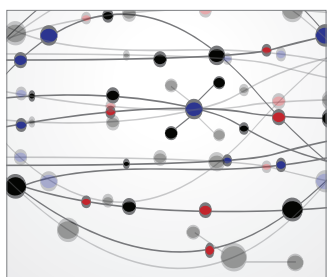

The Scientific World Journal
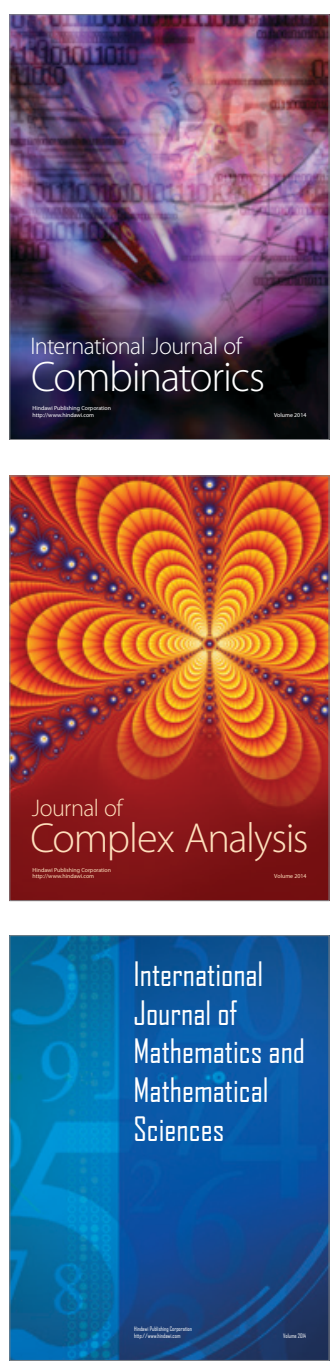
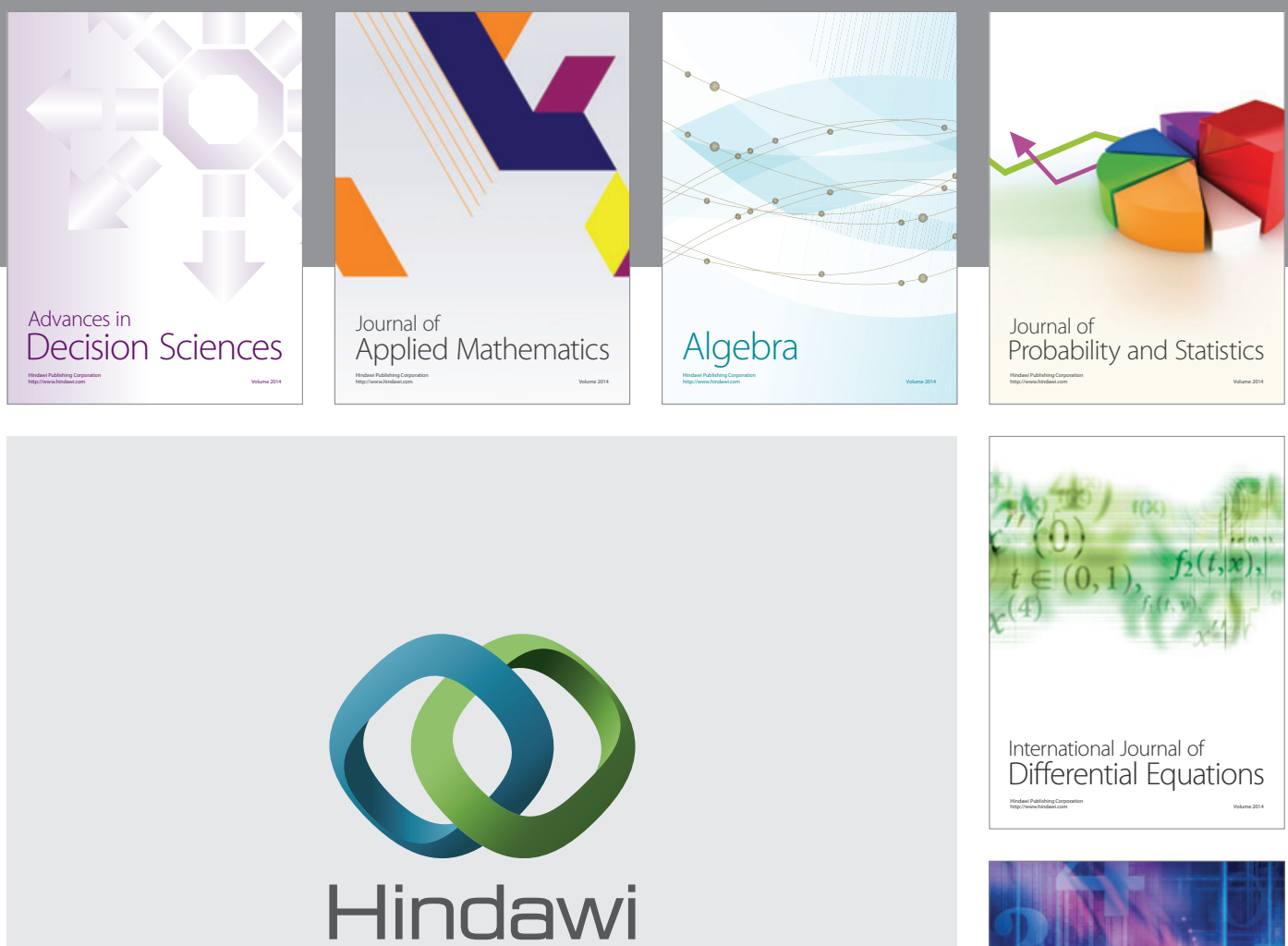

Submit your manuscripts at http://www.hindawi.com
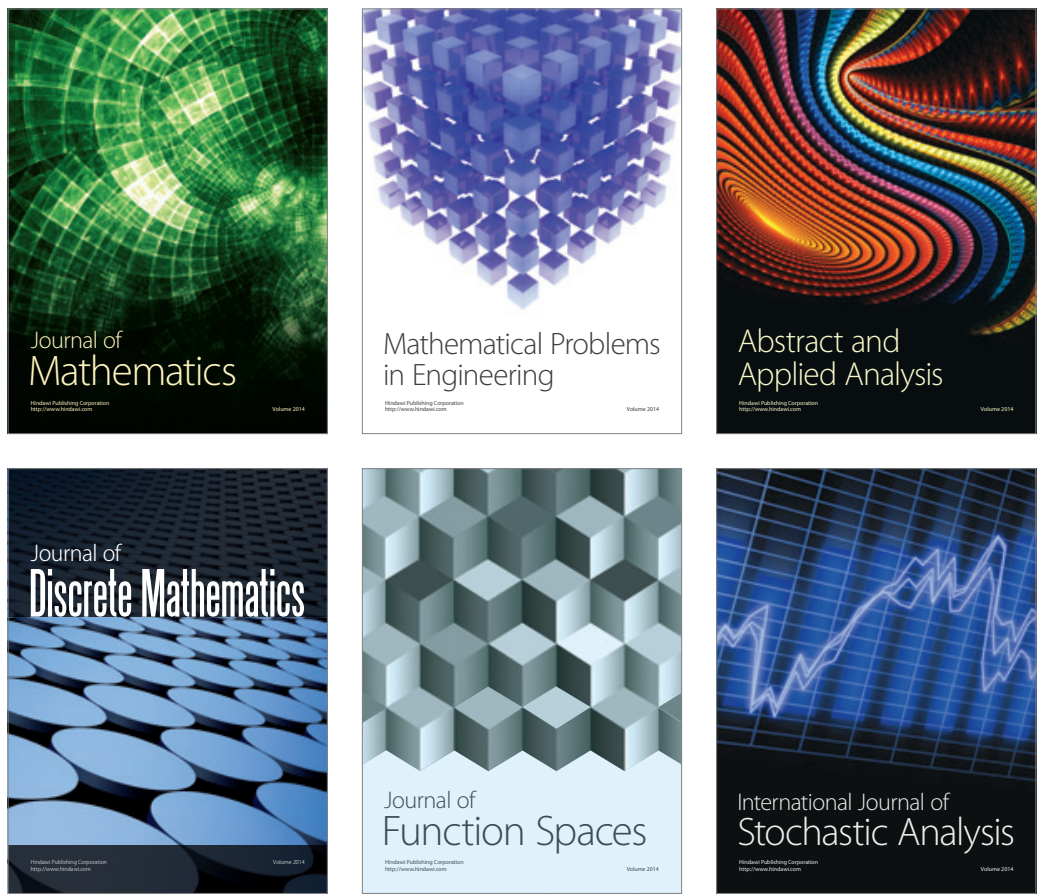

Journal of

Function Spaces

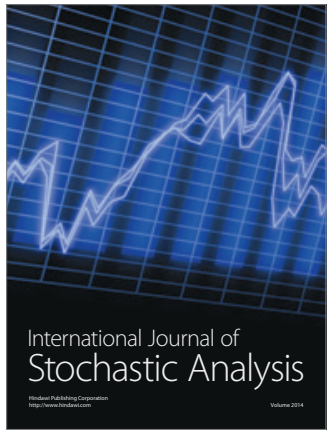

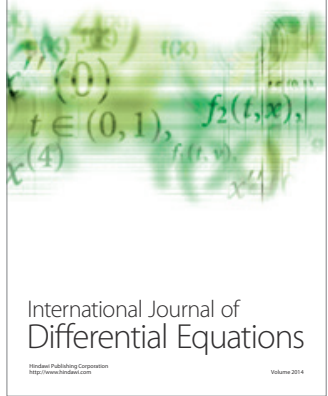
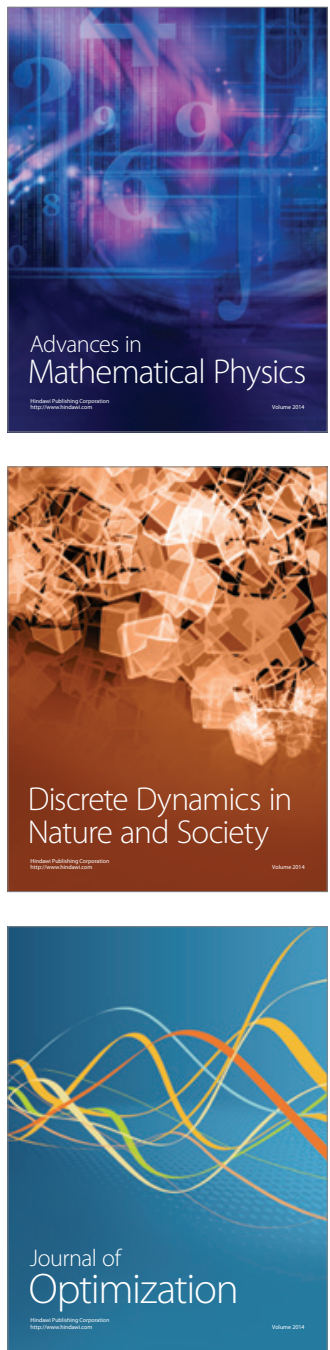